\section{Safety of endoscopic interventions in patients with thrombocytopenia}

\author{
Somashekar G. Krishna, Bhavana B. Rao, Selvi \\ Thirumurthi, Jeffrey H. Lee, Srinivas Ramireddy, \\ Michele Guindani, William A. Ross
}

Gastrointest Endosc 2014;80:425-34

Endoscopy and endoscopic interventions in patients with bleeding and coagulation disorders is a clinical dilemma due to lack of well-conducted studies. Many of the patients in our endoscopic practice have thrombocytopenia and the literature on the safety and efficacy of endoscopy is limited. Presence of low platelet count raises several questions like the lower limit of platelet count at which endoscopic pinch biopsy or endoscopic interventions can be performed safely or the efficacy of endoscopic hemostatic methods in patients with low platelet count. Some authors based on their personal experiences have suggested a minimum platelet count of 50,000 cells/cc for safe endoscopic procedures while others have advocated lowering the threshold to 20,000 cells/cc. ${ }^{[1-4]}$

The authors of this study performed a retrospective analysis of 395 consecutive patients ( 225 male, mean age \pm SD, 54.8 \pm 15.79 years) with platelet count of less than 50,000 cells/cc referred to their center for endoscopy. $84 \%$ of these patients had hematological malignancy with the most common indication for endoscopy being suspected gastrointestinal (GI) graft-versus-host disease (47.3\%) and GI bleed (GIB; $37.1 \%$ ) with $37 \%$ of endoscopies being done within 4 weeks of chemotherapy and/or hematopoietic stem cell transplantation. The platelet counts were obtained each morning in admitted patients and prophylactic platelet transfusions were given when platelet counts were less than 10,000 cells/cc. Furthermore, even if the counts were more than 10,000 cells/cc, but was thought to be low for endoscopy by either the primary oncology service or gastroenterology consultants, the patients were given platelet transfusions. All the endoscopies were performed by experienced faculty. A total of 617 endoscopic procedures including 351 upper GI endoscopy, 90 colonoscopies, and 176 sigmoidoscopies were performed.

Platelets were transfused within the $24 \mathrm{~h}$ before endoscopy in 329 patient encounters and 76 patient encounters $(23.1 \%$ of 329) were refractory to platelet transfusion. Endoscopic biopsy was done in $398(64.5 \%$ of 617$)$ procedures where the mean platelet count was $38.21 \pm 11.7 \times 103 / \mathrm{mL}$ and platelet transfusion was done in $65.8 \%$ procedures. Bleeding was seen in 6 endoscopies (1.5\%) (two upper GI endoscopy, two colonoscopies, and two flexible sigmoidoscopies) with it being immediate in five endoscopies. These five patients were treated with either endoclip placement (four patients) or epinephrine injection (one patient) whereas delayed bleeding in one patient was managed by endoclip placement. Of these six patients, one patient were given preprocedure platelet transfusion with pre- and post-transfusion platelet counts being 47,000 and 52,000 cells/cc respectively. The remaining five patients were not given preprocedure transfusion with mean platelet count of 38,400 cells/cc.

Forty five polyps were removed in 17 colonoscopies with mean platelet count of $39.65 \pm 8.53 \times 103 / \mathrm{mL}$ and platelets were transfused in 11 colonoscopies. Twenty-one polyps were removed with cold biopsy forceps biopsy, 19 with cold snare polypectomy, and 5 with hot snare polypectomy with only two polyps being more than $10 \mathrm{~mm}$ in size. The histological examination revealed hyperplastic in 5, tubular adenomas in 32 , tubulovillous adenomas in 6 , and sessile serrated adenomas in 2 patients with high-grade dysplasia in 3 polyps. Two patients had immediate bleeding with one patient undergoing hot snare polypectomy with a platelet count of 48,000 cells/ $\mathrm{cc}$ and the other undergoing cold forceps removal of polyp at a platelet count of 53,000 cells/cc. The bleeding was controlled by epinephrine injection with endoclips and gold probe respectively.

In patients with thrombocytopenia and GIB, angiodysplasias $(16.2 \%)$ and ulcers $(16.2 \%)$ were the most common causes of GIB. Endoscopic interventions (most commonly argon plasma coagulation) were performed in 41 endoscopies (active, 37; high-risk stigmata, 4) at a mean platelet count of $33.68 \pm$ $14.6 \times 103 / \mathrm{mL}$. Successful hemostasis was achieved in 39 endoscopies (95.1\%), and no adverse effects of the same were noted. The endotherapy failed in two endoscopies in one same patient with duodenal angiodysplasia done within a gap of 8 months. The preprocedure platelet count or platelet transfusion was not found to have any significant association with the risk of bleeding.

\section{Commentary}

This retrospective study has added significantly to the deficient literature in the field of endoscopy in patients with bleeding and coagulation disorders. The authors have demonstrated that in thrombocytopenic oncology patients, endoscopic procedures can be safely and effectively performed and the frequency of forceps biopsy and polypectomy related bleeding is elevated. Although the risk of bleeding is increased but in most of these patients the bleeding is minor, immediate, and therefore can be seen during the index procedure and easily controlled using endoscopic hemostatic methods. The authors have also found out that the pre-procedure platelet count could not predict the 
risk of GIB. These findings are in contrast to the current practice where endoscopic biopsies are usually not done at platelet counts lower than the 20,000 or 50,000 cells/cc and there is generally reluctance to perform endoscopic interventions in patients with thrombocytopenia questioning their efficacy in situations where normal hemostatic mechanisms of body are impaired. Also, it is important to remember that relying only on platelet counts is not correct as an important determinant of hemostasis is the platelet function and patients may bleed despite near normal platelet counts because of impaired platelet functions. The authors thus concluded that aiming for platelet counts of more than 50,000/cc before endoscopy is not necessary and platelet count more than $25,000 / \mathrm{cc}$ are sufficient for endoscopic biopsies, and platelet count more than 40,000 are reasonable for removal of small ( $<10 \mathrm{~mm}$ ) polyps with modest risk of bleed.

\section{Assessment of Crohn's disease activity by confocal laser endomicroscopy}

\begin{abstract}
Helmut Neumann, Michael Vieth, Raja Atreya, Martin Grauer, Jurgen Siebler, Thomas Bernatik, Markus F. Neurath, and Jonas Mudter
\end{abstract}

Inflamm Bowel Dis 2012;18:2261-2269

Endoscopy is the standard of care for diagnosing as well as assessing the extent and activity of inflammatory bowel diseases. ${ }^{[5,6]}$ The endoscopic changes visible on white light may lag behind the histopathological changes and therefore microscopic examination of the endoscopic biopsies is done to assess the histological activity. Confocal laser endomicroscopy (CLE) is a new upcoming diagnostic modality that has the potential of a real-time in vivo histopathology after application of exogenous fluorescence agent with a 1000 times magnification. ${ }^{[7]}$ CLE has been shown to be a useful diagnostic modality in ulcerative and microscopic colitis but its role in Crohn's disease $(\mathrm{CD})$ is not clear. ${ }^{[8,9]}$

In this prospective study 27 patients with quiescent and 27 patients with active CD were studied (mean age 39 years; $52 \%$ females) and were compared 18 controls (mean age: 68 years and $50 \%$ females). The CD activity in these patients was assessed using CD Endoscopic Index of Severity. The colonoscopy was performed by expert endoscopist and CLE was performed under conscious sedation using either an integrated endoscopy system (iCLE; Pentax) or a probe-based system (pCLE, Cellvizio, Mauna Kea Technologies, Paris, France). iCLE images were taken at a frame rate of $0.8 / \mathrm{s}$ at $1024 \times 1024$ pixels or $1.6 / \mathrm{s}$ at $1024 \times 512$ pixels. The normal mucosa and pathological lesions were evaluated as per Mainz Journal of Digestive Endoscopy Vol 5 | Issue 2 | April-June 2014 confocal pattern classification for iCLE and as per Miami classification for pCLE. ${ }^{[10,11]}$ After performing CLE, endoscopic biopsies were taken from both macroscopically normal and abnormal mucosa and findings of the confocal images were compared with the histology findings.

\section{Confocal Laser Endomicroscopy Findings in Controls}

All these controls had regular colonic architecture with normal crypts and microvascular pattern revealed normal vessel structures without fluorescein leakage or microerosions. There were no signs of increased cellular infiltrates within the lamina propria and the goblet cells were regularly shaped.

\section{Confocal Laser Endomicroscopy Findings in Quiescent Crohn's Disease}

Patients of CD had significantly increased number of colonic crypts (67\% vs. $11 \%$, respectively, $P=0.001)$, increased crypt tortuosity (52\% vs. $11 \%$, respectively, $P=0.02$ ), and dilated crypt lumens (52\% vs. $6 \%$, respectively, $P=0.008)$ compared with the controls. The frequency of increased vascularity $(44 \%$ vs. $6 \%$, respectively, $P=0.027)$ and an increased number of goblet cells (63\% vs. $6 \%$, respectively, $P<0.001$ ) was also more in patients of $\mathrm{CD}$ when compared to controls. However, there was no significant difference between the patients and controls in the presence of micro-erosions and cellular infiltrates within the lamina propria. Increased number of goblet cells on multivariate analysis was found to be the best predictor for quiescent CD (odds ratio, 28.9; 95\% confidence interval: 4.5-186.1).

\section{Confocal Laser Endomicroscopy Findings in Active Crohn's Disease}

The patients with active $\mathrm{CD}$ were found to be having significantly decreased number of colonic crypts (93\% vs. $6 \%$, respectively, $P<0.001)$, increased crypt tortuosity (100\% vs. $0 \%$, respectively, $P<0.001)$, dilated crypt lumen $(100 \%$ vs. $17 \%$, respectively, $P<0.001)$, more microerosions (100\% vs. $0 \%$, respectively, $P<0.001)$, increased cellular infiltrate $(100 \%$ vs. $0 \%$, respectively, $\mathrm{P}<0.001)$, increased vascularity ( $100 \%$ vs. $11 \%$, respectively, $P<0.001)$, and decreased goblet cells $(85 \%$ vs. $6 \%$, respectively, $P<0.001)$ in comparison to controls. The interobserver agreement ranged from moderate (crypt lumen, microerosions, goblet cell number) to substantial (tortuosity of crypts, vascularity) to almost perfect (number of colonic crypts, cellular infiltrate) with highest kappa-values for interobserver and intraobserver agreement being found for the number of colonic crypts ( 0.943 and 0.950 , respectively).

On the basis of these findings the authors proposed CD Endomicroscopic Activity Score (CDEAS) that consists of six parameters: Crypt number (increased or decreased), crypt distorsion, microerosions, cellular infiltrate, vascularity, and 